\title{
Numerical simulation of spray combustion of conventional fuels and Biofuels
}

\author{
A. E. Alajmi ${ }^{1}$, I. E. Abdalla ${ }^{1}$, T. Bengherbia ${ }^{1}$ \& Z. Yang $^{2}$ \\ ${ }^{1}$ Faculty of Technology, De Montfort University, UK \\ ${ }^{2}$ Department of Engineering and Design, University of Sussex, UK
}

\begin{abstract}
Numerical studies based on steady Computational Fluid Dynamics (CFD) for reactive flows were performed with the objective of validating advanced reaction mechanisms used to study spray combustion for both conventional and Biofuels. The SST-4 equation model was used to model turbulence, while more than one (comprehensive) reaction mechanisms were used to model the combustion of methanol, diesel and biodiesel using CHEMKIN-CFD and Fluent CFD code. Some of the reaction mechanisms used in modelling the current reactive flow simulation was already tested while others were developed during the course of this work. The computational results have shown good agreement with the available experimental data of Widmann and Presser (Combustion and Flame, 129, $47-86,2002)$ with the developed reaction mechanism slightly over predicting the temperature range. The CFD results have also shown that most of the harmful emission of the combustion of liquid fuels is less for Biodiesel compared to conventional diesel with the exception of $\mathrm{CO}_{2}$. This is in line with the finding of many experimental data.
\end{abstract}

Keywords: combustion, biofuels, emissions.

\section{Introduction}

Liquid fuels extracted from crude petroleum or produced from renewable sources (Biofuels) are predicted to remain the main sources of energy for long time to come. This is because other renewable energy sources such solar, wind, etc, has so far proved to be able to provide only a small fraction of the world energy, mainly in the developed world and with high cost (Dinica [2], Krozer [3]). Biofuels not only have the potential to replace conventional fuels, but also produce less emission 
of harmful gases compared to conventional fuels when burned under specific controlled environment such as Internal combustion Engines - ICEs (Enweremadu and Rutto [4], or other types of burners (Jaichandar and Annamalai [5]. However, for an optimal use by the existing combustion infrastructure (combustion chambers in ICEs or other burners), an effective combustion process is essential to ensure the maximum energy is extracted from Biofuels with a minimum impact on air quality. The rate of emission from burning conventional and Biofuels is a function of many parameters, some of them can be describes as macro-scale parameters (such as the equivalence ratio, $\Phi$ ) while others are associated with a much smaller features of the combustion process such as the chemical reactions and how they proceed under different temperature ranges and pressures.
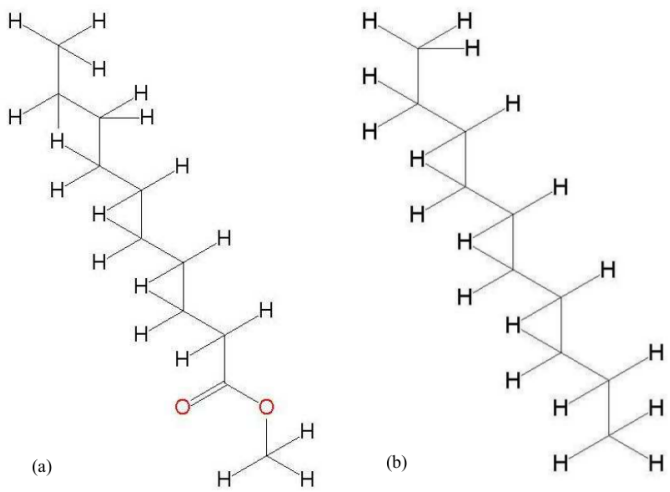

Figure 1: Chemical structure for (a) Methyl decanoate and (b) Decane.

A typical example of two hydrocarbons is shown in figure 1 where figure 1(a) is methyl decanoate $\left(\mathrm{C}_{11} \mathrm{H}_{22} \mathrm{O}_{2}\right)$, a Biofuel the closest conventional hydrocarbon to it is Decane $\left(\mathrm{C}_{10} \mathrm{H}_{22}\right)$, shown in figure 1(b). It is apparent that there is a difference between the two hydrocarbons not only in the number of Carbon and Hydrogen atoms, but also in the type of bonds. On top of this, the Biofuel (methyl-decanoate) descending from methyl esters has two Oxygen atoms in its composition. Therefore, one would expect that the way these hydrocarbons react and burn in a stream of air will differ. In order to adequately predict both the heat liberated and the rate of generation of the emission of Carbon oxides $\left(C O_{x}\right)$ and Nitrogen oxides $\left(N O_{x}\right)$ and other intermediate bi-products, it is essential to use the representative reaction mechanism to model most if not all the expected reactions in a combustion process.

Based on these arguments, the main aim of this manuscript is to present computational results for the combustion of different hydrocarbon (both conventional and Biofuels) using advanced reaction mechanism to shed light on the issues discussed in the above paragraph. The objectives behind the study can be summarised in two parts. The first is to develop, test and validate advanced 
reaction mechanism for the combustion of methanol used in the experimental studies of Widman et al. [1] and hence develop more mechanisms to model the combustion of other hydrocarbons that represent conventional and Biofuels. The second objectives is perform comprehensive analysis for the computational results and comment on the amount of energy liberated and emission produced from the combustion of conventional and Biofuels based on such advanced reactions mechanisms. The rest of this manuscript includes brief highlight of the method used, results and conclusion sections.

\section{Details of numerical computation}

The CFD code used to perform the current steady state calculations for the reactive flow described in this manuscript is Fluent 12.1 [6] coupled to CHEMKIN-CFD. Together, they facilitate the equilibrium and flamelet models for the combustion process. The equations governing flows with chemical reactions are the continuity, the species conservation equations and the energy. A solution to these equations provides in principle all the information sought from a reacting flow. The equations for the mean quantities in the Reynolds-averaged Navier-Stokes equation (RANS) approach are obtained by averaging the instantaneous governing equations using mass-weighted averages (Favre averages). The averaged governing equations can be written as follows:

- Conservation of Mass

$$
\frac{\partial \bar{\rho}}{\partial t}+\frac{\partial}{\partial x_{i}}\left(\bar{\rho} \widetilde{u}_{i}\right)=0
$$

- Conservation of Momentum

$$
\frac{\partial \bar{\rho} \widetilde{u_{i}}}{\partial t}+\frac{\partial}{\partial x_{i}}\left(\bar{\rho} \widetilde{u_{i}} \widetilde{u_{j}}\right)+\frac{\partial \bar{p}}{\partial x_{j}}=\frac{\partial}{\partial x_{i}}\left(\bar{\tau}_{i, j}-\bar{\rho} \widetilde{u_{i}^{\prime \prime} u_{j}^{\prime \prime}}\right)
$$

Where the viscous stress tensor $\tau_{i, j}$ for a Newtonian fluid and incompressible flow is given by

$$
\tau_{i, j}=2 \frac{\mu}{\rho} S_{i j}
$$

where $\mu$ is the laminar dynamic viscosity, and $S_{i j}$ is the strain rate tensor defined as:

$$
S_{i j}=\frac{1}{2}\left(\frac{\partial \widetilde{u_{i}}}{\partial x_{j}}+\frac{\partial \widetilde{u_{j}}}{\partial x_{i}}\right)
$$

- Conservation of Chemical species

$$
\frac{\partial\left(\bar{\rho} \widetilde{Y_{k}}\right)}{\partial t}+\frac{\partial}{\partial x_{i}}\left(\bar{\rho} \widetilde{u_{i}} \widetilde{Y_{k}}\right)=-\frac{\partial}{\partial x_{i}}\left(\overline{V_{k, i} Y_{k}}+\bar{\rho} \widetilde{u}_{i}^{\prime \prime} Y_{k}^{\prime \prime}\right)+\overline{\dot{\omega}}_{k}
$$

where $k=1,2, \ldots N$ species 
- Conservation of Energy

$$
\begin{aligned}
\frac{\partial \bar{\rho} \widetilde{h_{s}}}{\partial t}+\frac{\partial}{\partial x_{i}}\left(\bar{\rho} \widetilde{u_{i}} \widetilde{h_{s}}\right) & =\overline{\dot{\omega}}_{T}+\frac{\overline{D p}}{D t}+\frac{\partial}{\partial x_{i}}\left(\overline{\lambda \frac{\partial T}{\partial x_{i}}}-\overline{\rho u_{i}^{\prime \prime} h_{s}^{\prime \prime}}\right) \\
+\tau_{i, j} \frac{\partial u_{i}}{\partial x_{j}} & -\frac{\partial}{\partial x_{i}}\left(\rho \sum_{k=1}^{N} h_{s, k} Y_{k} V_{k, i}\right)
\end{aligned}
$$

where

$$
\overline{\frac{D P}{D t}}=\frac{\partial \bar{p}}{\partial t}+\overline{u_{i} \frac{\partial p}{\partial x_{i}}}=\frac{\partial \bar{p}}{\partial t}+\widetilde{u}_{i} \frac{\partial \bar{p}}{\partial t}+\overline{u^{\prime \prime} \frac{\partial p}{\partial x_{i}}}
$$

- Any Conserved scalar (such as mixture fraction)

$$
\frac{\partial}{\partial t}(\bar{\rho} \widetilde{Z})+\frac{\partial}{\partial x_{i}}\left(\bar{\rho} \widetilde{u}_{k} \widetilde{Z}\right)=\frac{\partial}{\partial x_{i}}\left(\widetilde{D}^{z}\right)-\frac{\partial}{\partial x_{i}}\left(\rho \widetilde{u_{k}^{\prime \prime} Z^{\prime \prime}}\right)
$$

This averaging procedure introduces unclosed quantities that have to be modelled. The detailed model used in this work is out of scope of this paper due to lack of space. Briefly, the Reynolds stresses term $\left(u_{i}^{\prime \prime} u_{j}^{\prime \prime}\right)$ was closed using Shear-Stress Transport (SST) $\mathrm{k}-\omega$ [7] model. As it is of interest to accurately predict pollutant and accounting for all the possible reaction pathways becomes and involve the maximum possible species and related reactions), a combustion model that allows detailed reaction mechanism to be used has to be selected. In this study, the Laminar Flamelet Model based on mixture fraction (Peters [8]) is used.
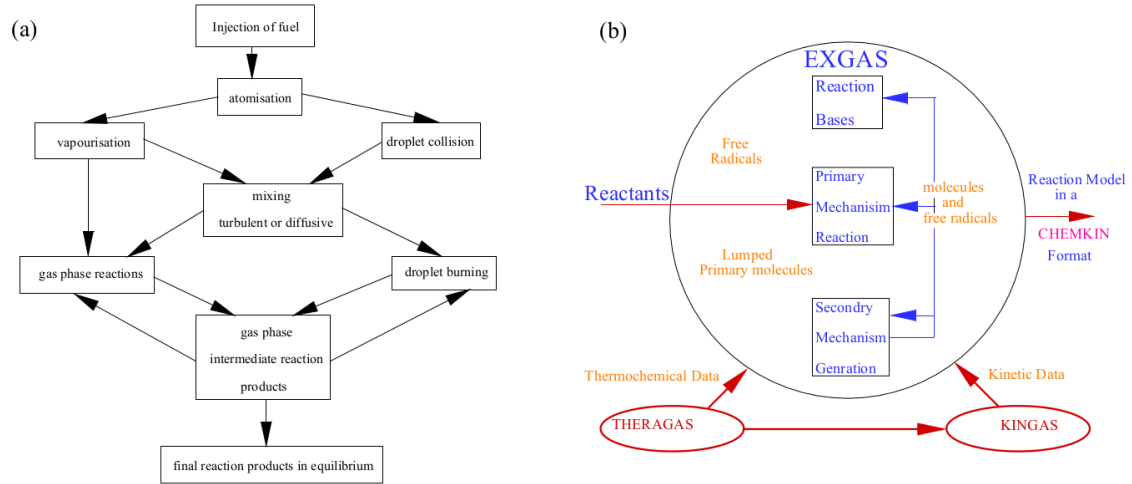

Figure 2: (a) Energy conversion cycle and (b) general description of the EXGAS system.

With the aid of the software Ex-Gas (Buda et al. [9]), best described by the flowchart in figure 2(b) and advanced and comprehensive reaction mechanism for a few conventional and Biofuels can be generated. The software connects three components and produces reaction mechanisms compatible with CHEMKIN-CFD 
for ANSYS FLUENT. This enable the flamelet model mentioned above to be used in addition to the equilibrium model. In addition, the well-known San Diego Mechanism [10] (referred to as SDM in the rest of this manuscript) is also used. For more documentation of the San Diego mechanism, the reader is advised to consult with [10]. Spray combustion is best explained in the flow chart of figure 2(a) which involve atomisation of the liquid fuel. The FLUENT's air-blast atomization model, which is a variation of the pressure-swirl model was used to model the atomisation of the liquid fuel used.

\subsection{Flow configuration, mesh and boundary conditions}

Shown in figure 3 is the computational domain (two top images) used in the simulations from which the results are presented below. Also shown the location of the probes Widmann and Presser [1] used to measure many parameters at the outlet of the burner. The simulation used two meshes, one of order 1 million and the second or order 2.5 million unstructured finite volumes generated using ICEM CFD. The mesh was stretched to resolve the wall shear layers, and the 2.5 million mesh has shown better flow resolution.

The no-slip boundary was applied to any solid surface while the opening boundary condition was used at the outlet. The opening boundary allows the flow to move in and out of the domain according to the pressure gradient generated by the flow field. In this situation, the flow moves out from the domain although in some few simulations, the outflow boundary has to be moved slightly up (figure 6(b)) to avoid such situation - happens on a negligible scale. The fuel flow rate into the burner was maintained at $3 \frac{\mathrm{kg}}{\mathrm{h}}$ and the air was admitted (at stoichiometric conditions) with a uniform velocity profile from the inflow boundary seen in figure 3 .

\section{Results and discussion}

The results presented in this paper are part of a few simulations that are conducted to simulate spray combustion for methanol, diesel and biodiesel. the simulations used different advanced combustion models and reactions mechanisms. Of interest to this paper the outcome of the reaction mechanism developed using Ex-Gas ( [9]) and modified using data from other sources such as Burcat [11]. This mechanism is referred to as EGM in the rest of this manuscript. The simulation for the combustion of Methanol was used for validating the computational results relying on Widmann and Presser [1] while the simulation of Diesel and Biodiesel are part of the project at hand.

\subsection{Validation of the computational results}

Shown in figure 4(a) is the comparison of temperature profiles that exactly matches the 13 measurement point for the experimental data of Widmann and Presser [1]. The results were obtained using flamelet model with SDM for Methanol fuel. 

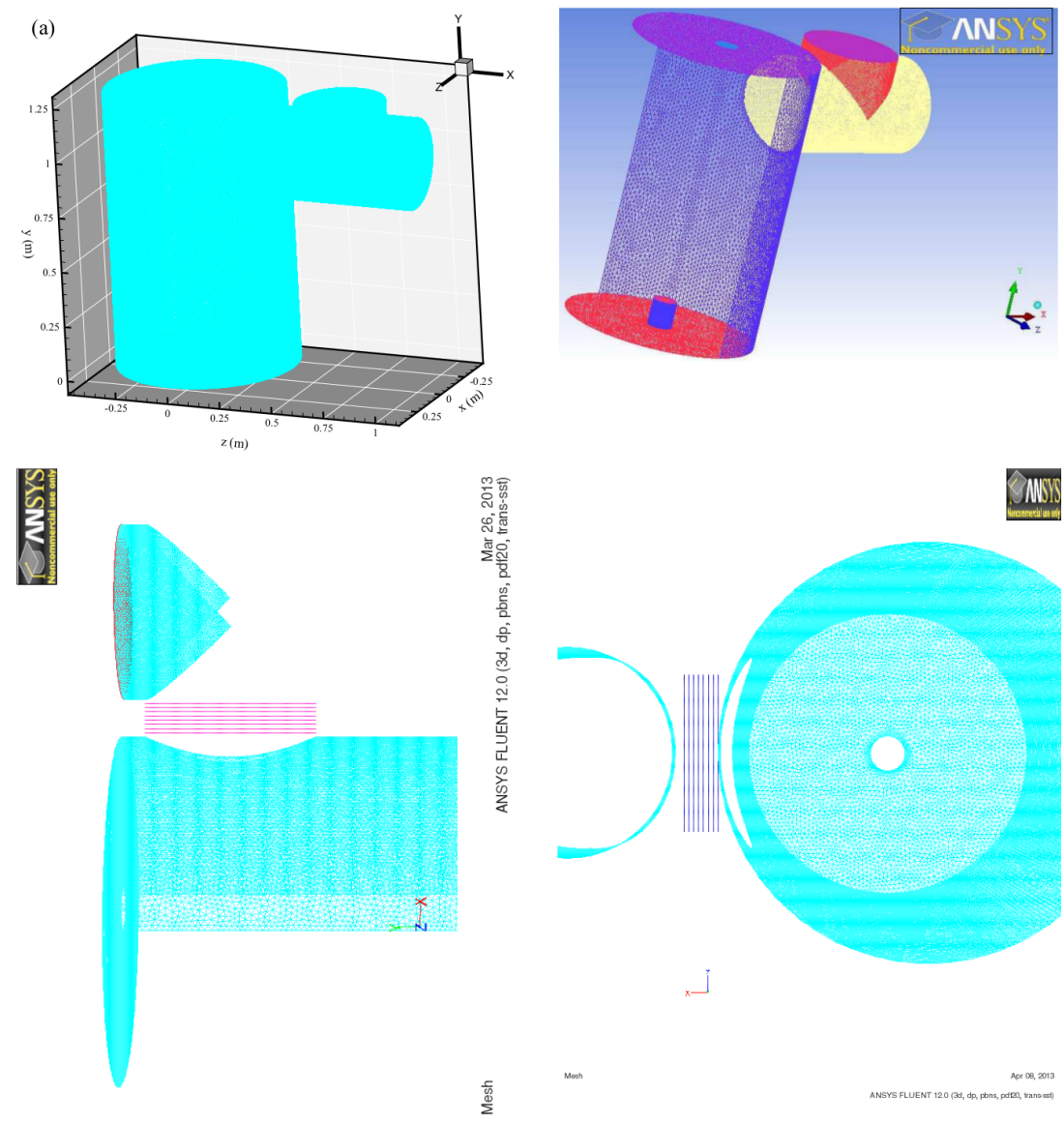

Figure 3: Computational geometry and location of the vertical and horizontal probes according to Widmann and Presser [1].

It is apparent that the CFD results agree very well with the computation results while using the well tested SDM. This in principal indicates that the CFD problem set-up for the spray combustion is relevant and the results indeed present a realistic outcome. Worth to mention that there large number of parameters in these simulations that requires correct set up at the start of the simulation to ensure adequate results.

The 8-vertical lines shown in figure 3 for the SDM simulation are shown in figure 4(b) while the simulation for liquid methanol using the developed EGM is shown in figure 4(c). It is clear that whilst the SDM predict more or less the same temperature range of the experimental data of Widmann and Presser [1], the results based on the EGM slightly over predicted the temperature distribution at locations that corresponds to the experimental data. However, the difference is still marginal 

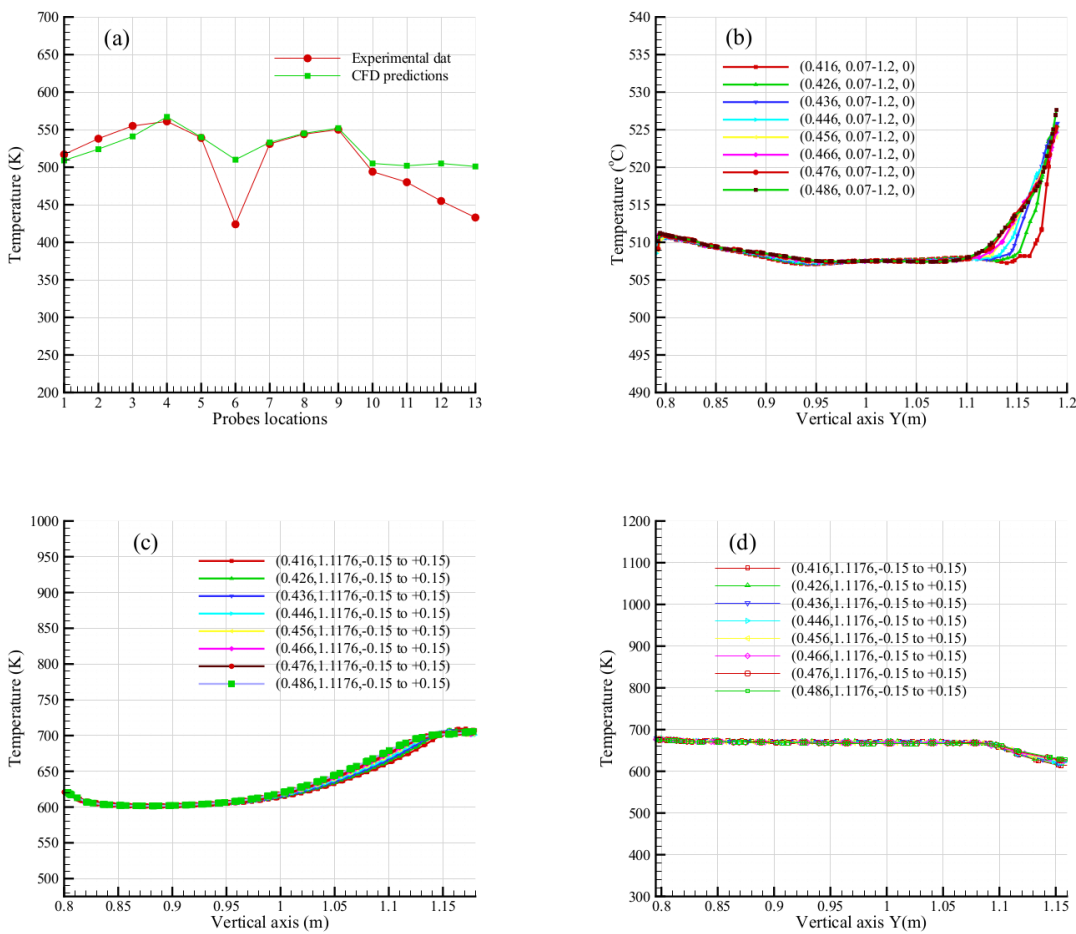

Figure 4: Temperature distribution along the vertical probe lines: (a) comparison with experimental results; (b) vertical lines for SDM - methanol; (c) vertical lines for EGM - methanol; (d) vertical lines for EGM - Diesel.

(of order 10-15\%). To explain the reason behind this difference, one need to bear in mind that while the number of species and reactions used in SDM are kept to the minimum, a total of 206 reactions and 77 species are used in the Exgas mechanism. This renders the SDM to a type of reduced reaction mechanism. It is apparent that whilst it is an advantage to use as much species and reactions in a mechanism, the simulator need to understand the complexity of these reactions and their paths and rates. Whilst those who developed the EGM software may have explained few points, the experience of this work proved that much more is needed in order to comment on any developed reaction mechanism. Having said so, the author of this manuscript is not in favour of criticising the outcome of the EGM results as it might be very correct and the experiment, which was conducted more than a decade ago, may have its own drawbacks. In fact Widmann and Presser [1] have mentioned a specific error percentages in their results.

In comparison to Methanol's simulations of figures 4(b) and (c), figure 4(d) shows the temperature profiles for the vertical locations using Diesel (approximated as Dodecane $\mathrm{C}_{12} \mathrm{H}_{26}$ ). The simulation was performed based on 

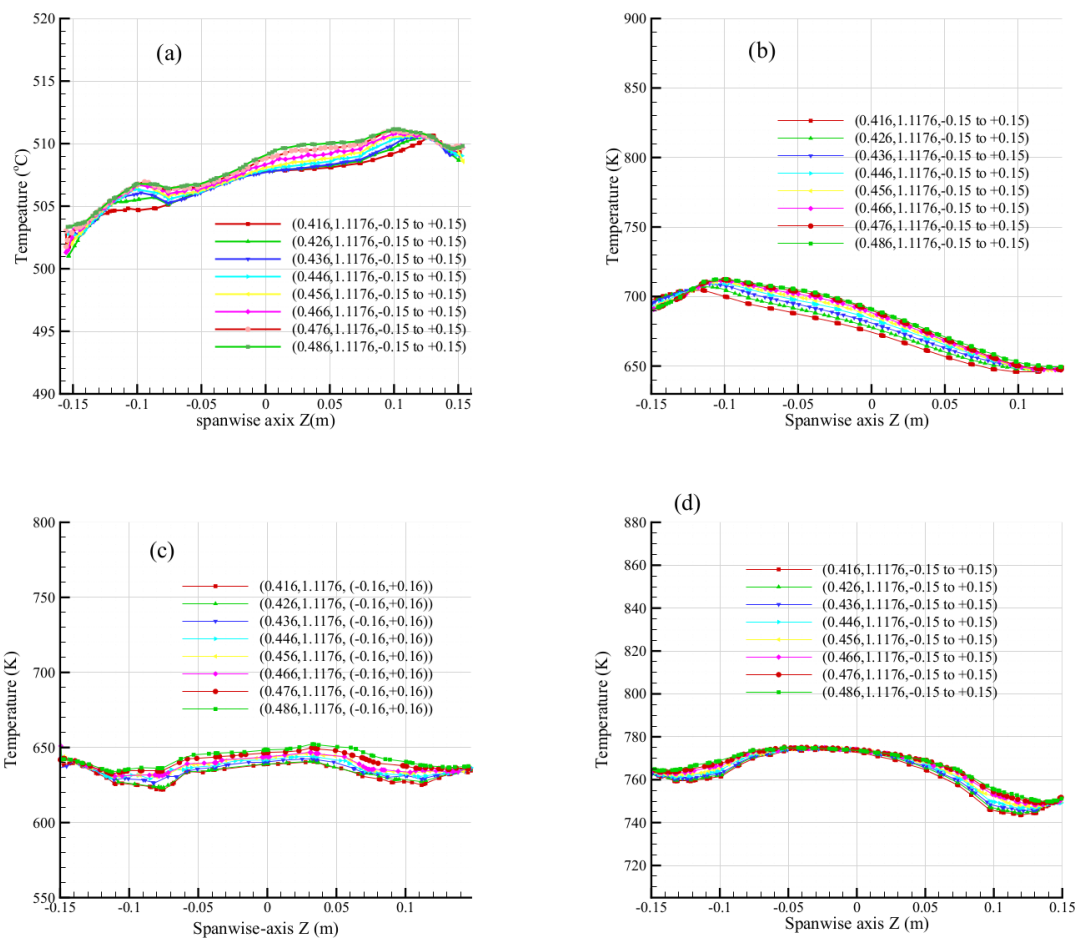

Figure 5: Temperature profiles along the horizontal probe lines: (a) SDM methanol; (b) EGM - methanol; (c) EGM - Diesel; (d) EGM - BioDiesel.

the Equilibrium model and thermal data generated from EGM. The temperature profile for this fuel is higher than that predicted for Methanol using both EGM and SDM. The reasons could be partially attributed to the energy content difference between the two fuels with Diesel higher in energy and burning it may indeed leads to higher temperature range. In this sense the CFD simulation results is reliable, however, one difficulty that faced the results here was that upon using the flamelet model with both Diesel and Biodiesel, the temperature range is significantly higher (of order $800 \mathrm{~K}$ ) and the author is still exploring whether this is realistic or this is attributed to the EGM comprehensive reaction mechanism developed for these fuels.

The profiles for the horizontal lines shown in figure 5 which corresponding some experiment probe locations are show in figures 5(a) for SDM Methanol; (b) for EGM Methanol; (c) for EGM Diesel and (d) for EGM Biodiesel approximate as Methyl Decanoate $\left(\mathrm{C}_{11} \mathrm{H}_{22} \mathrm{O}_{2}\right)$. Methyl Decanoate was chosen as the Biofuel close to Diesel (Dodecane). It is noticeable that the CFD result for both Diesel and Biodiesel predicts higher temperature range with Biodiesel having the highest range (order $760 \mathrm{~K}$ compared to $700 \mathrm{~K}$ for Diesel and $500 \mathrm{~K}$ for Methanol). Many 

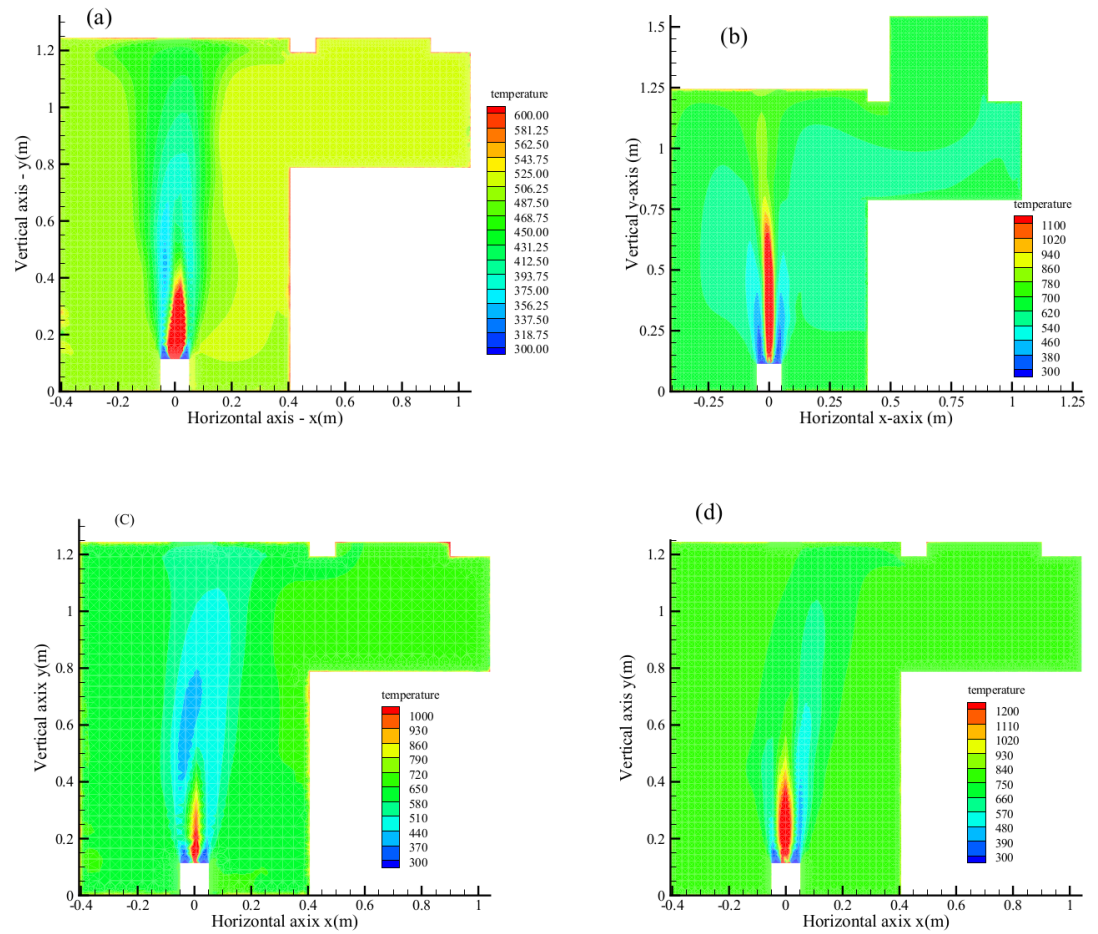

(d)

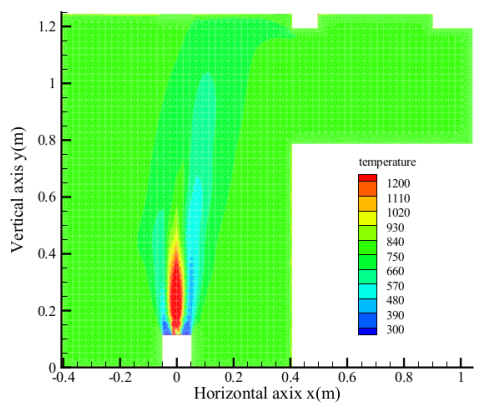

Figure 6: Temperature distribution along a central plane: (a) SDM - Methanol; (b) Exgas mechanism - Methanol; (c) Exgas mechanism - Diesel; (d) Exgas mechanism - BioDiesel.

experimental work on Engines has shown that Diesel has higher energy content $(10-15 \%)$ than biodiesel and hence predicting higher temperature for Biodiesel compared to diesel may not fit in this energy framework. Therefore, beyond any doubt the reason behind this is down to the reaction mechanism used although the equilibrium model was used for both Diesel and Biodiesel in this study. Again this finding indicate that using a comprehensive mechanism to model the combustion of large hydrocarbon such as Dodecane and Methyl Decanoate needs much more detailed investigation.

\subsection{Flame structure}

A central slice for the temperature is shown in figure 6 for Methanol using the SDM (a) and EGM (b) while the Diesel and Biodiesel were shown in figures 6(c) and (d) respectively. The figures give an indication to the flame structure under the distinct simulation conditions and it is apparent that the EGM provide the most 

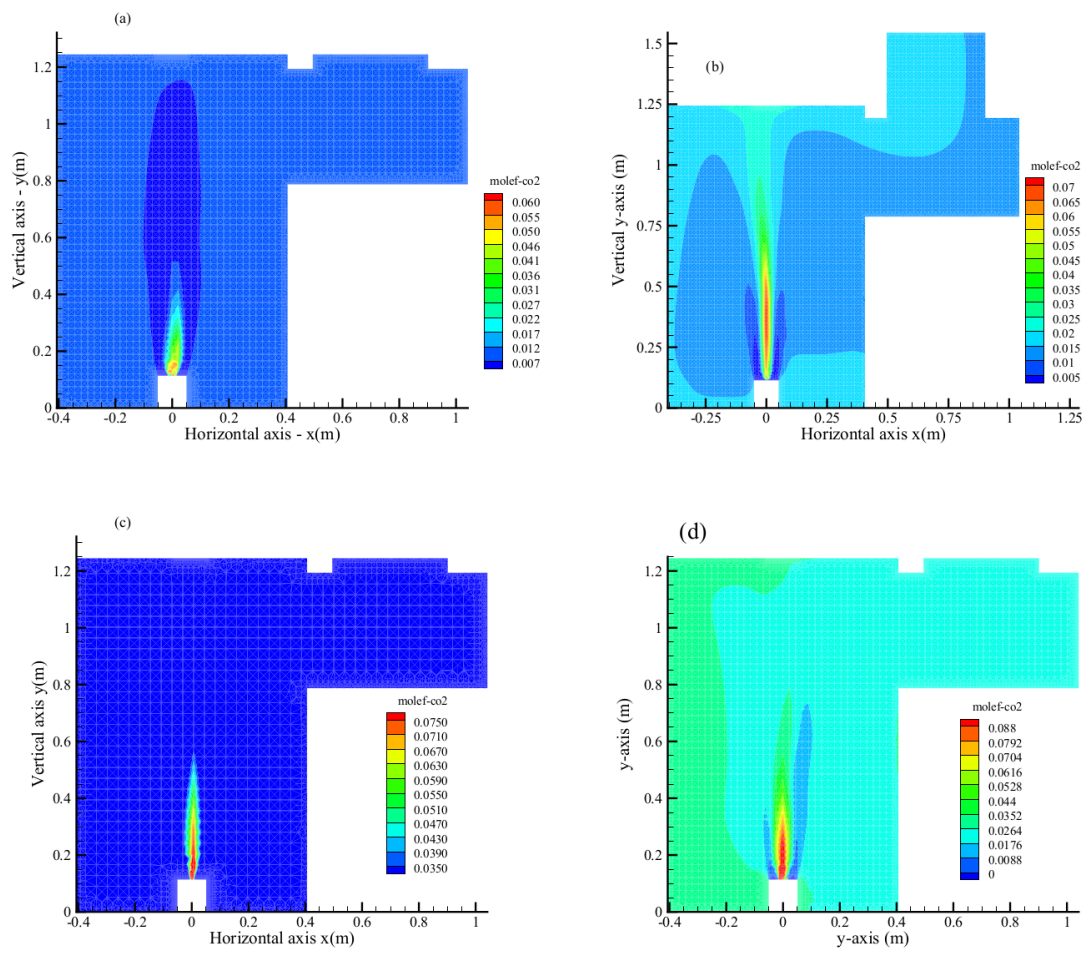

Figure 7: $\mathrm{CO}_{2}$ profiles along the horizontal probe lines: (a) SDM - methanol; (b) EGM - methanol; (c) EGM - Diesel; (d) GM - BioDiesel.

establish flame structure although going into details is out of scope for this short paper.

\subsection{Predicted pollutants rates}

One of the objectives of this work is to examine the prediction of pollutants using the different combustion models and reaction mechanisms in this study. A central slice showing the contours for $\mathrm{CO}_{2}$ for the 4 simulations mentioned above is showing in figure 7. It is apparent that the EGM simulation of Methanol using the flamelet model produced the most detailed distribution of the $\mathrm{CO}_{2}$ compared to the rest. One could attribute this to the fact that the reaction mechanism used here is more detailed than the rest of the three simulations, one advantage of using such mechanisms. The results also show that Biodiesel produces the highest rate of $\mathrm{CO} 2$ under the same air/fuel ratio, a fact that is supported by a few experimental works in this area. 


\section{Conclusion}

A comprehensive reaction mechanism for Methanol, Diesel and biodiesel was generated and tested using the experiment of Widmann and Presser [1] as a benchmark. The computational results showed good agreement with the experimental data; however, the author acknowledges the complexity of modelling reactive flow using large number of species and reactions. whilst it is an advantage to use the most comprehensive reaction mechanism possible, the simulator need to exercise some cautious and develop more understanding to the possible routes of the reactions involved and the rate of generation of different intermediate species and how such species affect the path of the combustion process. In other words, the reaction mechanism used may play a vital role in predicting accurate results in terms of temperature and pollutants rates

\section{References}

[1] Widman, J.F. and Presser, C.A. Benchmark experimental database for multiphase combustion model input and validation. Combustion and Flame, 129, 47-86, 2002.

[2] Dinica, V. Renewable electricity production costs - A framework to assist policy-makers' decisions on price support. Energy Policy 39 pp. 4153-4167, 2011.

[3] Krozer, Y. Cost and benefit of renewable energy in the European Union. Renewable Energy 50, pp. 68-73, 2011.

[4] Enweremadu, C.C., Rutto, H.L. Combustion, emission and engine performance characteristics of used cooking oil biodiesel. Renewable and Sustainable Energy Reviews, Volume 14, Issue 9, December 2010, pp. 2863 2873, 2010.

[5] Jaichandar, S., Annamalai, K. Effects of open combustion chamber geometries on the performance of pongamia biodiesel in a DI diesel engine. Fuel, Volume 98, pp. 272-279, 2012.

[6] Ansys CFX version 12.1, www.ansys.com/cfx (accessed 20 February, 2013).

[7] Menter, F.R. Two-equation eddy-viscosity turbulence models for engineering applications, AIAA Journal, Vol. 32, No. 8, pp. 1598-1605, 1994.

[8] Peters, N. Laminar Diffusion Flamelet Models in Non-Premixed Turbulent Combustion. Progress in Energy and Combustion Science, 10, pp. 319-339, 1984.

[9] Buda, F., Bounaceur, R., Warth, V., Glaude, P.A., Fournet R. and F. BattinLeclerc. Combust. Flame 142 (2005), pp. 170-186.

[10] http://web.eng.ucsd.edu/mae/groups/combustion/mechanism.html. Date of access: 01/09/2012.

[11] http://garfield.chem.elte.hu/Burcat/burcat.html. Date of access: 01/08/2012. 